\title{
Dynamic Restoration With GMPLS and SDN Control Plane in Elastic Optical Networks [Invited]
}

\author{
Alessio Giorgetti, Francesco Paolucci, Filippo Cugini, and Piero Castoldi
}

\begin{abstract}
Optical transport networks typically deploy dynamic restoration mechanisms in order to automatically recover optical connections disrupted by network failures. Elastic optical networks (EONs), currently emerging as the next-generation technology to be adopted in optical transport, introduce new challenges for traditional generic multiprotocol label-switching (GMPLS)-based restoration that may seriously impact the achievable recovery time. At the same time, the software-defined networking (SDN) framework is emerging as an alternative control plane. It is therefore important to investigate possible benefits provided by SDN in the implementation of restoration mechanisms for EONs. This paper proposes a dynamic restoration scheme for EONs based on the SDN framework. The proposed scheme contemporarily exploits centralized path computation and node configuration to avoid contentions during the recovery procedure with the final aim of minimizing the recovery time. The performance of the proposed scheme is evaluated by means of simulations in terms of recovery time and restoration blocking probability and compared against three reference schemes based on GMPLS and SDN.
\end{abstract}

Index Terms-Control plane; Elastic optical network (EON); GMPLS; PCE; Provisioning; Restoration; SDN.

\section{INTRODUCTION}

$\mathbf{E}$ lastic optical networks (EONs) are evolving from wavelength-switched optical networks (WSONs) in the transport segment of current communication networks [1,2]. In EONs the bandwidth of optical links is organized in a flexible grid using a number of $12.5 \mathrm{GHz}$ frequency slices [3]. The flexible grid allows the utilization of optical connections (i.e., lightpaths) with variable bandwidth occupancy that can be implemented by a single optical carrier or by a number of subcarriers that are jointly routed from source to destination in the optical domain (i.e., super chan$n e l)$ [1]. This bandwidth flexibility enables the utilization of advanced modulation formats, thus leading to higher spectral efficiency and increasing the amount of supportable traffic.

Manuscript received July 1, 2014; accepted October 10, 2014; published November 11, 2014 (Doc. ID 214025).

A. Giorgetti (e-mail: a.giorgetti@sssup.it), F. Paolucci, and P. Castoldi are with the TeCIP Institute, Scuola Superiore Sant'Anna, Pisa 56124, Italy.

F. Cugini is with Consorzio Nazionale Inter-universitario per le Telecomunicazioni (CNIT), Pisa 56124, Italy.

http://dx.doi.org/10.1364/JOCN.7.00A174
To enable EON operation several upgrades are required in the data plane (e.g., multiflow transponders, bandwidthvariable nodes) and in the control plane (e.g., support of bandwidth-variable lightpaths) of currently deployed WSONs. Moreover, due to dynamic arrival/departure of lightpaths with different bandwidth occupation, spectrum fragmentation typically emerges in EONs after some operation time. Since the fragmentation issue can significantly degrade the network efficiency, several solutions have been proposed such as in-line or planned defragmentation procedures. These solutions require the utilization of a centralized controller storing all the information regarding the established lightpaths $[\underline{4}, \underline{5}]$. Therefore, a control plane implementing a set of network functionalities in a centralized manner is currently envisioned as the most suitable solution for EONs.

Two alternatives are currently in discussion for the implementation of an EON control plane: a GMPLS/PCE control plane where the distributed generic multiprotocol label-switching (GMPLS) protocols are assisted by a stateful and active path-computation element (PCE) devoted to path computation and spectrum defragmentation [6] and a control plane based on the emerging software-defined networking (SDN) framework fully relying on a centralized controller that, besides path computation and spectrum defragmentation, is also in charge of lightpath establishment and management (e.g., recovery) [7].

Scalability and reliability are the typical issues regarding the implementation of a centralized control plane. Scalability concern is more related to other network scenarios (e.g., Internet Protocol (IP)/MPLS networks) where a single network can include thousands of nodes, whereas EONs are typically composed of less than a hundred nodes. Conversely, control-plane reliability is crucial in EONs. However, there are several proposals of possible distributed implementations of logically centralized SDN control planes [ $[\underline{8}, \underline{9}$. These works typically propose the utilization of a set of communicating controllers, each one managing a portion of the whole network and improving both scalability and reliability. Therefore, at this stage, there is a certain convergence toward the utilization of an SDN-based logically centralized control plane for EONs.

Several works recently explored the potentials of SDN showing that the main functionalities supported by the GMPLS/PCE control plane can be implemented using 
SDN [10]. However, until now, there has not been clear evidence about whether SDN is able to provide benefits with respect to GMPLS/PCE. Specifically, SDN has been demonstrated to provide shorter lightpath setup time [11]. However, setup time is not a critical parameter during provisioning when a new lightpath has to be activated. Conversely, the time needed to recover a lightpath that has been disrupted by a network failure (i.e., recovery time) is a critical parameter because it has a direct impact on service-level agreement (SLA) satisfaction. For instance, optical tunnels, network virtualization, and data-center interconnection are typical services to be provided by EONs, all requiring fast and effective recovery techniques. Therefore, it is important to quantify the benefits that a SDN control plane can provide during restoration with respect to a GMPLS/PCE control plane.

Besides spectrum fragmentation, EONs introduce new challenges during restoration with respect to traditional WSONs that may seriously impact the achievable recovery time. First, the fine granularity of the flexible grid potentially increases the number of lightpaths that can be disrupted by a single link failure [12]. Second, the configuration of EON nodes using spectrum selective switches (SSSs) based on liquid-crystal-on-silicon (LCoS) technology requires longer times, i.e., several tens of milliseconds $[13,14]$. Both the aforementioned problems increase the contention probability among the numerous signaling sessions typically triggered upon network failure, leading to further recovery attempts and, finally, increasing the recovery time [15]. Therefore, during recovery, the utilization of a centralized SDN controller that is able not only to coordinate the computation of backup paths but also the actual establishment of computed backup paths could provide significant benefits.

This paper proposes a novel restoration scheme for EONs based on the SDN framework and compares its performance against distributed GMPLS restoration, GMPLS/ PCE restoration, and a straightforward implementation of SDN restoration. The proposed scheme bundles the signaling messages needed to establish the backup paths in fewer messages to synchronize the needed node reconfigurations and, therefore, minimize the recovery time. Such an approach is not compatible with the GMPLS control plane where an independent source-driven signaling session is always required for each lightpath to be recovered also if a PCE is used for path computation.

The rest of this paper is organized as follows: Section II reviews the previous work on EONs controlled by GMPLS and SDN frameworks. Section III details the issues arising in the implementation of restoration in EONs and describes the considered restoration schemes. Section IV describes the simulation scenario and presents the obtained results. Finally, Section V concludes the paper.

\section{PRevious Works}

Dynamic restoration in GMPLS-based WSONs is a well-investigated topic that has been widely covered by theoretical, simulation, and experimental studies [15-17].
However, the implementation of such techniques in EONs, where typical node-configuration time is significantly higher with respect to WSONs $[13,14]$, may imply increased recovery time due to possible contentions among different signaling sessions [15]. This paper proposes and evaluates the implementation of dynamic restoration using the SDN frameworks that are currently emerging as alternative control planes for WSONs and EONs [10].

Most of those SDN frameworks are based on the OpenFlow protocol $[18,19]$ that is used for communications between node-located agents (i.e., OpenFlow switches$\mathrm{OSs}$ ) and the centralized controller (i.e., OpenFlow controller-OC).

The first applications of OpenFlow in the context of WSONs are reported in $[\underline{13}, \underline{20}-22]$. The works in $[20,21]$ propose an OpenFlow-based control plane for packetand circuit-switched networks including experimental demonstrations. The authors of $[13,22]$ focus on WSONs. Specifically, the work in [13] demonstrates an OpenFlowbased control plane supporting lightpath setup and release; the work in [22] presents an integration of OpenFlow and GMPLS protocols. In this case, the OC does not directly configure the optical nodes but communicates with the GMPLS controller of the lightpath source node that starts the lightpath setup using RSVP-TE signaling.

More recently, a number of works focused on lightpath provisioning $[23,24]$ and physical impairments evaluation $[25,26]$ in EONs using an SDN control plane. Moreover, a comprehensive demonstration of an SDN control plane has been provided in [27], which also considers recovery mechanisms. Those works mainly demonstrated that SDN can be used to manage and dynamically control EONs; however, a comparison against the GMPLS/PCE control plane is not explicitly provided.

Our previous works in $[28,11]$ perform a comparison of the SDN control plane and the GMPLS/PCE control plane. Specifically, [11] only focuses on the provisioning phase, whereas [28] performs an initial analysis of the restoration phase indicating that bundling of signaling messages is effective in reducing the recovery time within a centralized control plane. This paper extends the work in [28] with extensive simulation results considering multiple values of node-configuration and path-computation times, as well as the provisioning scenario. Moreover, this work includes in the comparison a fully distributed GMPLS control plane, which is still considered the most promising solution for the implementation of dynamic restoration [29].

\section{Restoration IN EONs}

Independent of the utilized control plane, dynamic restoration is triggered in EONs after the detection of a network failure. After failure detection the control plane is typically subject to a high number of almost simultaneous backup path requests that have to be established in the shortest possible time to recover the disrupted traffic. Therefore, contentions among different signaling sessions are likely 
during restoration and very detrimental because they strongly degrade the recovery time.

Specifically, two kinds of contentions may take place: spectrum contentions (i.e., a lightpath attempting to reserve a frequency slice previously reserved by another lightpath on the same link) and node-configuration contentions (i.e., a lightpath attempting to configure a node that is currently undergoing another configuration). Spectrum contentions typically happen in fully distributed scenarios (i.e., GMPLS) where the computation of backup paths is independently performed by the source node of each disrupted lightpath considering a locally stored trafficengineering database (TED). Several solutions have been proposed to alleviate this problem in WSONs [15,30]. However, those solutions are not considered in the standard GMPLS recovery procedure, which in the case of spectrum contention applies the crankback procedure to trigger further signaling attempts (using different spectrum slots) and thus significantly increases the recovery time [31,32]. Node-configuration contentions are almost negligible in WSONs, where nodes based on microelectromechanical systems (MEMS) technology typically require a configuration time of few milliseconds [33]. Conversely, their effect can be relevant in EONs where nodes based on LCoS technology typically require a configuration time of several tens of milliseconds $[13,14]$. Indeed, during restoration, a high number of signaling messages are asynchronously received at network nodes. In this context, the required node configurations triggered at each node by the received messages are queued, implying significant recovery time degradation.

Second, the configuration of EON nodes using SSSs based on LCoS technology requires longer times, i.e., several tens of milliseconds [13,14].

The rest of this section describes four restoration schemes to be applied in EONs deploying GMPLS or SDN control planes. Specifically, Subsection III.A describes the standard restoration procedure using a fully distributed GMPLS control plane. Subsection III.B describes a restoration scheme based on the GMPLS control plane where a centralized PCE is used for computing the backup paths. Subsection III.C describes two restoration schemes based on the SDN control plane. The first scheme (i.e., SDNind) is the reference SDN restoration procedure, and the second (i.e., SDN-bund) is our proposal that jointly exploits the centralized path computation and the bundling of signaling messages to avoid both spectrum and nodeconfiguration contentions.

\section{A. GMPLS Restoration}

With a fully distributed GMPLS control plane, link failures are detected by the node attached to the disrupted link and advertised on the network using OSPF-TE link-state advertisements (LSAs) and/or RSVP-TE notify messages. Upon notification reception, the failure recovery is initiated by the source node triggering the following steps: 1) network resources used by the disrupted lightpath are released, sending an RSVP-TE Tear message along the disrupted path; 2) a backup path is locally computed, bypassing the disrupted link; and 3) an RSVP-TE Path message is sent along the computed route up to the destination node that selects the spectrum slot to be used and replies to the source node with a RSVP-TE Resv message, actually reserving network resources along the backup path (i.e., performing node configuration).

Since the aforementioned procedure is independently executed for each disrupted lightpath, both spectrum contentions and node-configuration contentions are likely in GMPLS restoration.

Figure 1 represents a network scenario where two lightpaths (i.e., $L 1$ and $L 2$ ) have been disrupted by the failure of link 1-4. L1 was originally routed on the working path 1-4 and $L 2$ was originally routed on the working path $0-1-4$. The backup path of $L 1$ is $1-2-4$, whereas the backup path of $L 2$ is $0-3-4$. Figure 2 illustrates a typical architecture of an EON node where SSSs are used in both the switching fabric and the add/drop modules [34]. In particular, Fig. 2 refers to node 4 of the network scenario in Fig. 1 where both the recovered lightpaths require a reconfiguration of the SSSs in the first add/drop module. This is a typical situation that generates a node-configuration contention.

Figure 3(a) illustrates the recovery procedure using the GMPLS scheme in the network scenario represented in Fig. 1. A node-configuration contention occurs at node 4; indeed, when the message Path(L2) reaches node 4 a hardware configuration is already ongoing as an effect of the previously received message Path(L1). In this case, the hardware configuration required to recover L2 is queued until termination of the previous configuration, and the recovery of L2 is consequently delayed.

\section{B. GMPLS/PCE Restoration}

If a PCE is used the recovery procedure changes only at step (2). Indeed, the path computation is not performed by the source node of each disrupted lightpath, but delegated to the centralized PCE. Specifically, the PCE protocol (PCEP) is utilized by the source nodes to send a backup

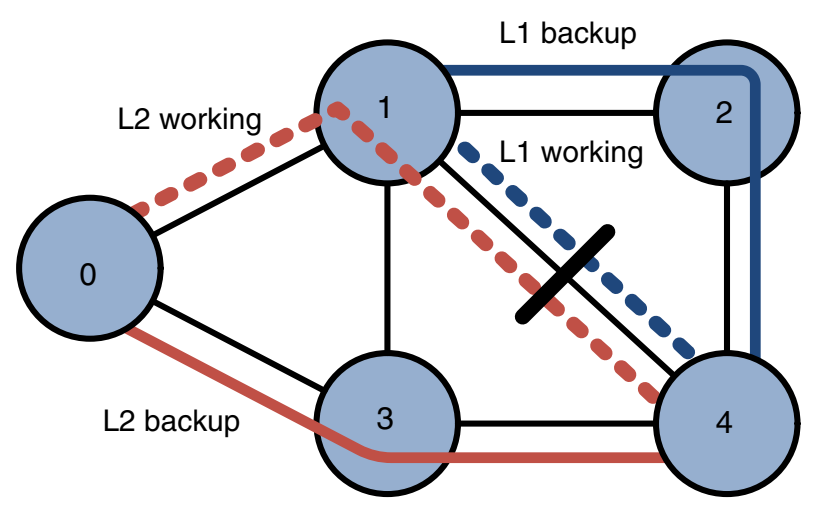

Fig. 1. Example of network failure. The failure of link 1-4 disrupts the working paths of two lightpaths, $L 1$ and $L 2$. 


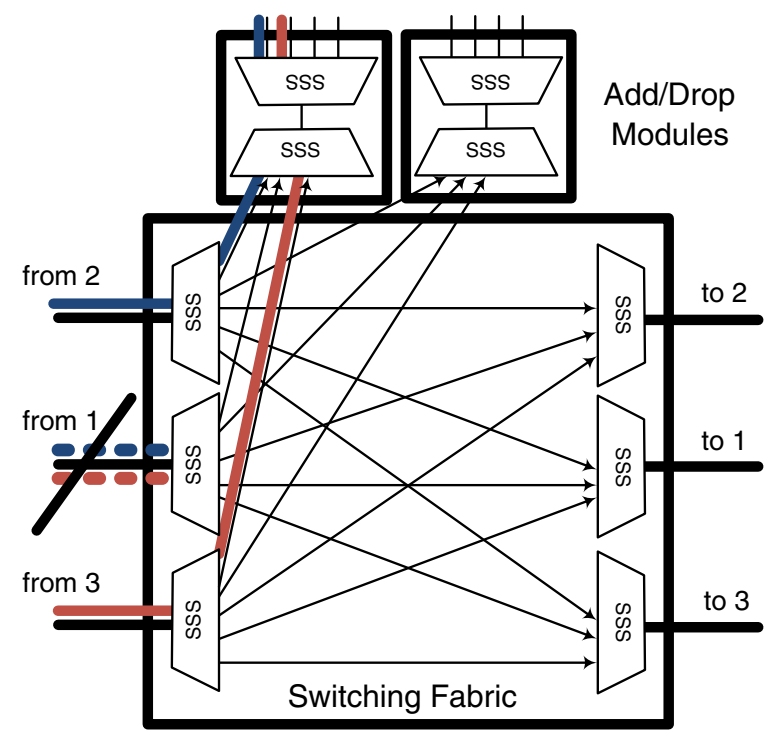

Fig. 2. Typical architecture of an EON node with nodal degree 3 and 2 add/drop modules. Lightpaths and node IDs refers to node 4 of Fig. 1.

path computation request to the $\mathrm{PCE}$ and to receive the related reply. Once the backup path is received from the PCE, the RSVP-TE signaling is triggered to actually reserve the required resources.

Since all backup paths are computed at the PCE considering the same TED, the applied routing and spectrum assignment scheme can be designed so that spectrum contentions are almost avoided $[35,36]$. Conversely, nodeconfiguration contentions are still likely in this scenario because the signaling sessions required for establishing the several backup paths are independently triggered for each disrupted lightpath. Moreover, this scenario introduces two other issues that could degrade the recovery time. First, the PCEP communication introduces a further delay. Second, since all path computations are queued at the PCE, the path-computation time becomes a critical parameter to be considered.
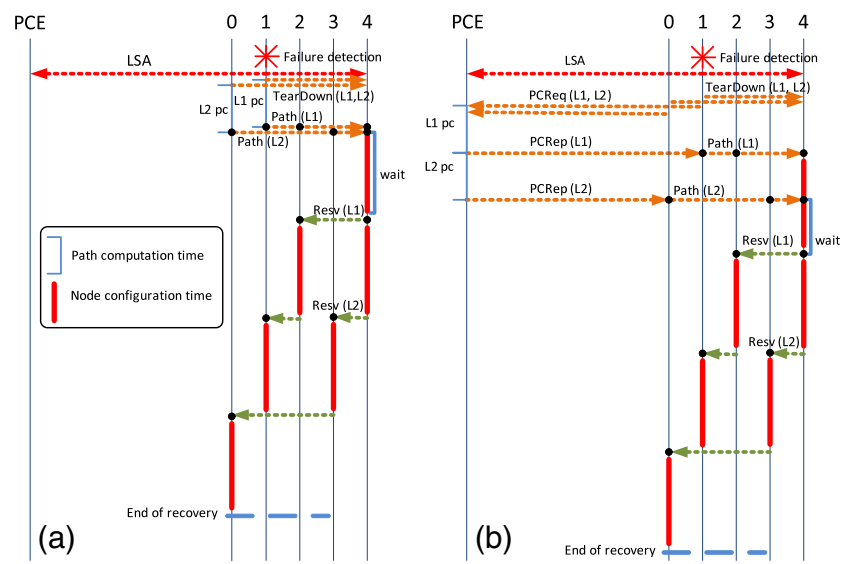

Figure 3(b) illustrates the recovery procedure using the GMPLS/PCE scheme in the network scenario represented in Fig. 1. The figure shows that due to the PCEP communication and to the path-computation queuing at the PCE the beginning of the RSVP-TE signaling is delayed with respect to the GMPLS case. Moreover, the node-configuration contention is still present at node 4 .

\section{SDN Restoration}

Using an SDN-based control plane the detecting node directly informs the OC through an OpenFlow PORT STATUS message. The OC drives the recovery procedure triggering the following steps: 1) the list of the disrupted lightpaths is computed using the stored lightpath database; 2) resources occupied by disrupted lightpaths are locally freed in the TED; and 3) backup path computation is performed and TED is updated, occupying resources used by the computed backup path.

After the aforementioned steps, the computed backup paths have to be actually established in the network. Specifically, two OpenFlow-based schemes are hereafter described for backup path configuration: the independent restoration scheme (SDN-ind) and the bundle restoration scheme (SDN-bund).

1) SDN-ind Scheme: Using this scheme the OC triggers an independent OpenFlow communication after each backup path computation using a number of FLOW MOD messages sent, in parallel, to each OS traversed by the backup path. FLOW MOD messages configure the new flow entries required for the computed backup path and delete the flow entries previously used by the disrupted path.

Figure 3(c) illustrates the recovery procedure using the SDN-ind scheme in the network scenario represented in Fig. 1. In this case the signaling to recover each disrupted lightpath is performed in parallel so that the total recovery time is expected to be considerably reduced with respect to the GMPLS cases. However, since a different signaling session is utilized for each disrupted lightpath,
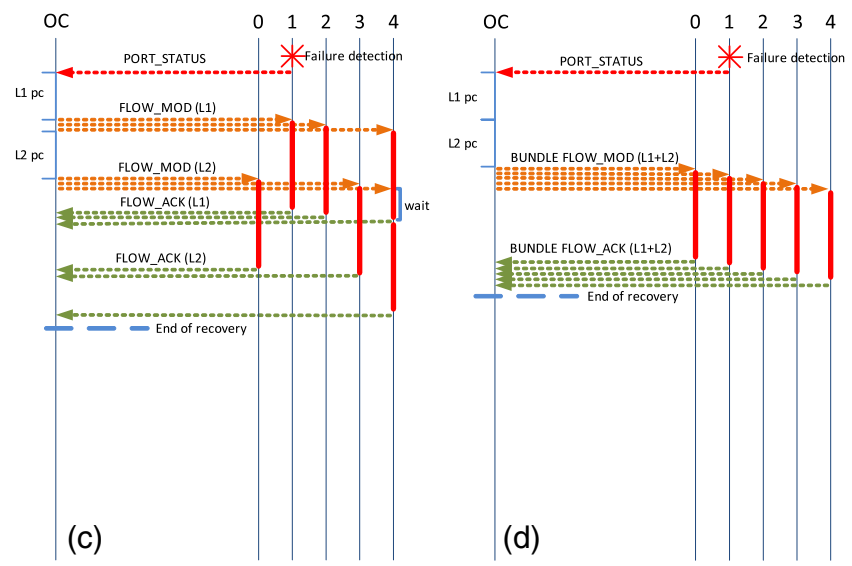

Fig. 3. Signaling timeline of the four considered restoration schemes: (a) GMPLS restoration, (b) GMPLS/PCE restoration, (c) SDN-ind restoration, and (d) SDN-bund restoration. 
node-configuration contentions may still occur as illustrated at node 4 in Fig. 3(c).

2) SDN-bund Scheme: Using this scheme the OC executes the backup path computation for all the disrupted lightpaths before triggering the OpenFlow signaling. When the backup path computation is terminated, the OC sends a bundle message (i.e., BUNDLE FLOW MOD) to each involved node. The BUNDLE FLOW MOD message, first proposed in [28], encloses all the component FLOW MODs, related to different lightpaths, accounting for all the reconfiguration required at the specific node. The possibility of creating bundle messages, including multiple configurations to be performed by the network node, has also been introduced in the latest version of the OpenFlow specification [18]. The node receiving the BUNDLE FLOW MOD modifies all the local flow entries, then performs a single node reconfiguration. This multiple configuration is allowed by current node-software tools, and the configuration time is not dependant on the number of filter modifications to be enforced.

Figure 3(d) illustrates the recovery procedure using the SDN-bund scheme in the network scenario represented in Fig. 1. The figure shows that by avoiding nodeconfiguration queuing delays at node 4 the recovery time can be further reduced.

\section{Performance Evaluation}

The aim of the simulation study described in this section is to compare the considered restoration schemes in terms of blocking probability and lightpath setup time during both the provisioning and the restoration phases. With specific focus on the lightpath setup time it is of particular interest to understand the effect of the signaling parallelization typical of both the considered SDN schemes, and the effect of the node-configuration contention avoidance introduced by the proposed SDN-bund scheme.

\section{A. Simulation Scenario}

The described schemes are evaluated by means of simulations using a custom-built event-driven $\mathrm{C}++$ simulator. The considered Pan-European network topology, illustrated in Fig. 4, includes 27 nodes and 55 bidirectional links with 256 frequency slices of $12.5 \mathrm{GHz}$. Traffic is uniformly distributed among node pairs, and lightpaths arrive following a Poisson process. Two types of lightpath requests are considered: $100 \mathrm{Gbps}$ requests are served with a single optical carrier using three frequency slices and 400 Gbps requests are served with a super-channel using ten frequency slices [12].

The four schemes are evaluated using the same routing and spectrum assignment (RSA) algorithm during both provisioning and restoration phases. In particular, routing is performed considering a set of candidate paths $P_{s, d}$ that for each node pair $(s, d)$ includes all the paths within one hop from the shortest. Using TED, the path with the largest number of available frequency slots capable of

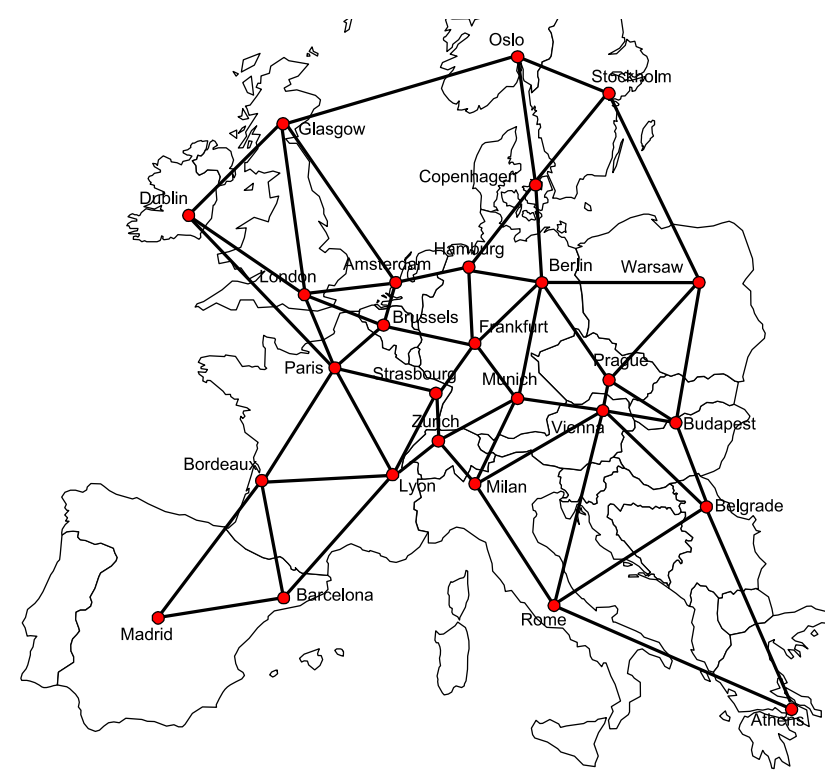

Fig. 4. Test network topology.

accommodating the lightpath is selected. Spectrum assignment is first-fit.

Achieved results are plotted with the achieved confidence interval, at $90 \%$ confidence level. Packets processing time at each node has been derived from experiments performed using Juniper M10 routers supporting the RSVPTE protocol. The obtained value is $10 \mu$ s for those packets that are just forwarded, and $2 \mathrm{~ms}$ for those packets requiring a local processing (e.g., RSVP-TE path). If not differently specified typical values are assumed for the time required to perform a path computation, either at the PCE or at the source node (i.e., $10 \mathrm{~ms}[37,38]$ ), and for the time required to perform a single node configuration (i.e., $50 \mathrm{~ms}[\underline{13}, \underline{14}]$ ).

\section{B. Simulation Results: Provisioning}

During provisioning the average holding time of lightpath requests is kept fixed at one hour. The network load is varied by changing the average interarrival time. These assumptions implement a typical trade-off between static and dynamic traffic conditions that, besides the effect of traffic load, allow to evaluate also the effect of outdated network state information $[\underline{15}, \underline{39}]$.

Figure 5 depicts the achieved provisioning blocking probability under variable network load. The provisioning blocking probability is defined as the ratio between the blocked lightpath requests and the overall requested lightpaths. In accordance with previous results in [15], the figure shows that the GMPLS scheme, not using a centralized path computation, exhibits a blocking floor due to spectrum contentions for low and medium loads. Conversely, the other schemes achieve very similar blocking, only emerging for high traffic loads. 


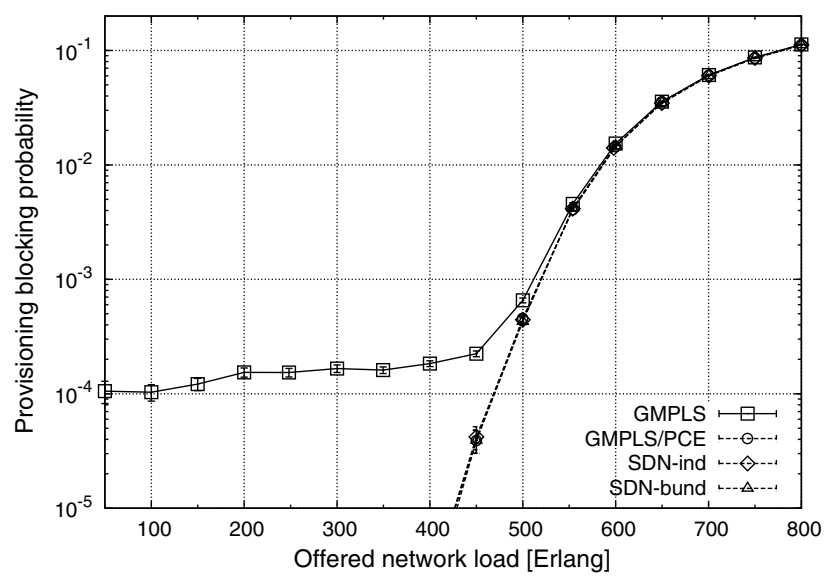

Fig. 5. Provisioning blocking probability.

Figure 6 depicts the achieved average provisioning time under variable network load. The provisioning time is defined only for effectively established lightpaths as the time between the generation of the lightpath request and the conclusion of the related signaling session. The figure shows that the introduction of an SDN control plane is able to significantly reduce the setup time due to the parallelization of the signaling process. Conversely, the figure shows that during provisioning the effect of nodeconfiguration avoidance is negligible; in fact, SDN-ind and SDN-bund schemes achieve the same setup time. Finally, the figure shows that the GMPLS/PCE scheme has only a slight setup time increase with respect to the GMPLS case. This increase is due to the required PCEP communication between the source nodes and the PCE.

\section{Simulation Results: Restoration}

The restoration results have been obtained by simulating a number of independent link failures (i.e., at least 2500) per simulation point. Each failure affects a single

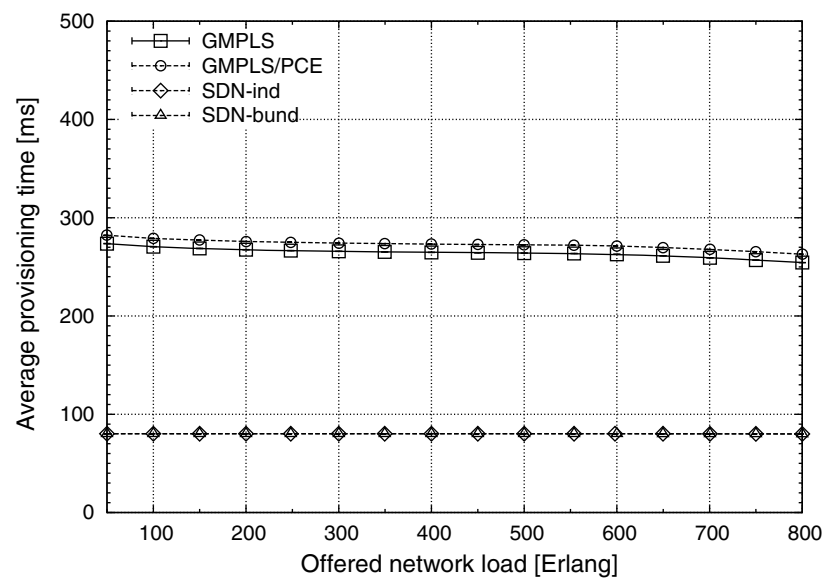

Fig. 6. Average provisioning setup time: path-computation time is $10 \mathrm{~ms}$ and node-configuration time is $50 \mathrm{~ms}$.

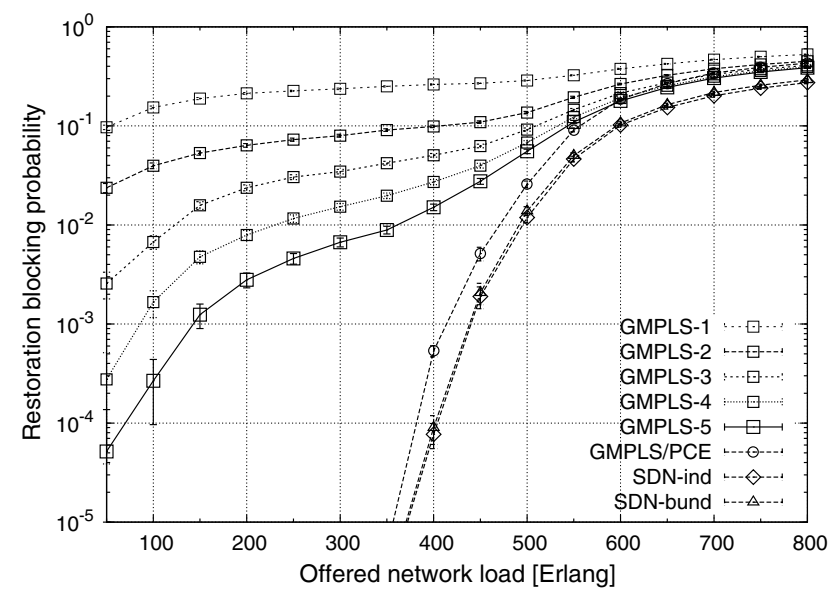

Fig. 7. Restoration blocking probability.

data plane bidirectional link, assuming that the control plane remains fully operational. Before starting the generation of failures the network is provisioned at the desired load. In this case, different network loads are obtained by changing the average holding time of lightpath requests, whereas the average interarrival time is kept fixed at $10 \mathrm{~s}$.

Figure 7 shows the restoration-blocking probability under variable network load. Restoration-blocking probability, for each failure, is defined as the ratio between the unrecovered lightpaths and the lightpaths disrupted by the failure. Specifically, the blocking achieved with the GMPLS scheme is depicted after one, two, three, four, and five crankback attempts. Conversely, for the other schemes only the blocking probability achieved after one attempt is plotted. The figure shows that the blocking achieved by GMPLS after five crankback attempts is significantly higher than the blocking achievable after a single attempt using a centralized computation. This is mainly due to spectrum contentions that are very common when the recovery is managed in a distributed way. The other three schemes achieve similar blocking, but the GMPLS/PCE scheme provides a slight degradation with respect to SDN schemes due to residual spectrum contentions that may occur during signaling sessions.

Figure 8 depicts the average recovery time under variable network load. The recovery time is defined only for effectively recovered lightpaths as the time between the failure and the conclusion of the signaling used to establish the backup path. The figure shows that the recovery time is strongly reduced by the proposed SDN-bund scheme. Specifically, avoiding queuing of node configurations, SDNbund achieves a recovery time of about $120 \mathrm{~ms}$, almost independent of the network load. Conversely, all other schemes exhibit a recovery time that is increasing with the network load up to high loads where the recovery time stabilizes due to high restoration-blocking probability. Moreover, Fig. 8 shows that the GMPLS scheme provides higher recovery time with respect to GMPLS/PCE. This result is not straightforward and demonstrates that the utilization of a PCE coordinating the recovery procedure to avoid spectrum contentions is able to supply for the delay introduced by the PCEP communication (see Fig. 3). Indeed, 


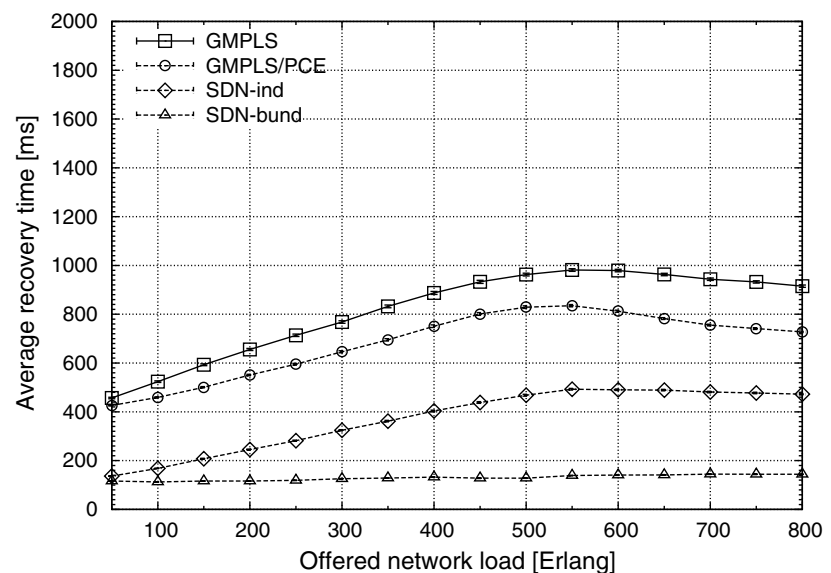

Fig. 8. Average recovery time: path computation time is $10 \mathrm{~ms}$ and node configuration time is $50 \mathrm{~ms}$.

the distributed path computation utilized in the GMPLS scheme typically implies multiple crankback attempts, finally leading to higher values of the average recovery time.

However, the results in Fig. 8 strongly depend on the considered path-computation time and on the considered node-configuration time. Therefore, it is important to analyze the effect of the aforementioned parameters on the average recovery time.

Figure 9 depicts the average recovery time as a function of the path-computation time. The figure shows that there is benefit in using a centralized element for performing the path-computation during recovery (i.e., the PCE or the OC) only if the path-computation time is under a specific threshold. Indeed, the recovery time of GMPLS is almost independent of the path-computation time because it parallelizes the path computations among network nodes, whereas other schemes exhibit a linear increase of the average recovery time because all path computations are queued at the controller. In particular, GMPLS/PCE provides benefit with respect to GMPLS up to a path computation time of about $35 \mathrm{~ms}$. SDN-ind provides benefit up to $70 \mathrm{~ms}$. SDN-bund provides benefit up to $150 \mathrm{~ms}$.

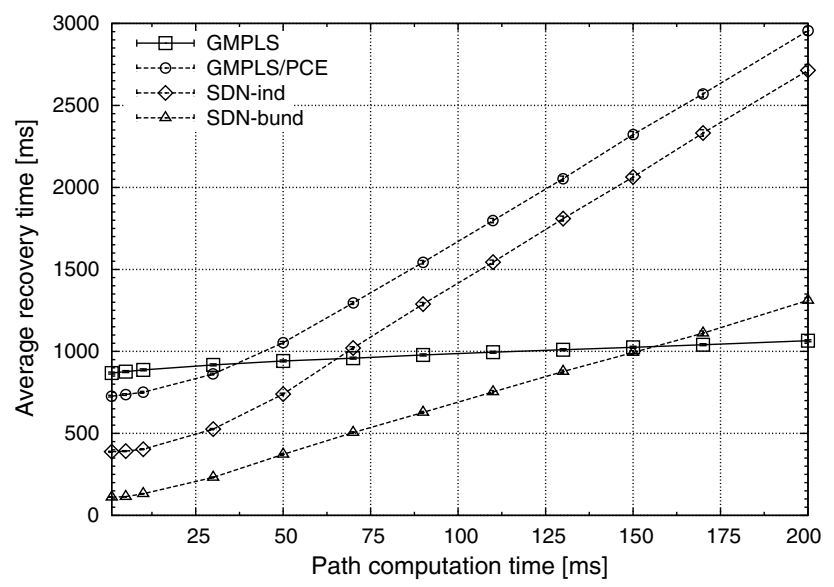

Fig. 9. Average recovery time: node configuration time is $50 \mathrm{~ms}$.

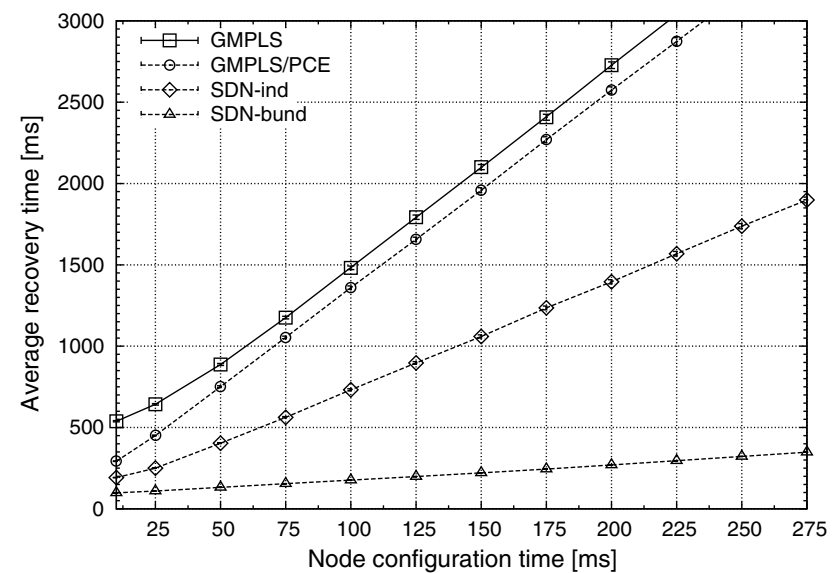

Fig. 10. Average recovery time: path computation time is $10 \mathrm{~ms}$.

Path-computation time is quite variable, depending on the considered algorithm, ranging from a few milliseconds to several tens of milliseconds. However, $100 \mathrm{~ms}$ has been demonstrated to be sufficient for performing advanced path computation including physical impairment validation [38]. Therefore, the results in Fig. 9 demonstrate that a centralized approach during restoration can provide significant benefit with realistic values of the pathcomputation time, especially if it is associated with avoidance of node-configuration contentions (i.e., SDN-bund scheme).

Figure 10 depicts the average recovery time as a function of the node-configuration time. The figure shows that with all the considered schemes, the average recovery time linearly increases with the node-configuration time. However, the obtained slopes are very different; specifically, the GMPLS case is the less scalable solution when node configuration times typical of EONs are considered (i.e., higher than $100 \mathrm{~ms}$ ) [14]. Conversely, the proposed SDN-bund scheme is the most scalable in terms of node-configuration time because node-configuration contention avoidance is particularly important for high values of the nodeconfiguration time.

\section{Conclusion}

A novel scheme supporting provisioning and restoration in EONs has been proposed using the SDN framework (i.e., SDN-bund). Its performance has been compared against GMPLS, GMPLS/PCE, and a straightforward implementation of SDN (i.e., SDN-ind).

Simulation results showed that provisioning and restoration-blocking probabilities are considerably reduced if a centralized path computation is utilized. Moreover, the provisioning results showed that, thanks to the parallelization of the signaling process, both SDN schemes reduce the lightpath setup time with respect to GMPLS-based schemes. Finally, restoration results demonstrated that, under several realistic network scenarios, the proposed SDNbund scheme effectively combines the centralized path 
computation with the bundled node configuration to significantly reduce the lightpath recovery time.

Future work is planned on this topic to validate effectiveness and feasibility of the proposed schemes in a logically centralized scenario using multiple controllers to improve control-plane scalability and reliability.

\section{ACKNOWLEDGMENTS}

This paper is an extended version of the work presented in [28]. This work was partially funded by the European Community's Seventh Framework Programme FP7-2007-2013 through the Integrated Project (IP) IDEALIST under grant agreement num. 317999.

\section{REFERENCES}

[1] M. Jinno, H. Takara, Y. Sone, K. Yonenaga, and A. Hirano, "Multiflow optical transponder for efficient multilayer optical networking," IEEE Commun. Mag., vol. 50, no. 5, pp. 56-65, May 2012.

[2] S. Frisken, S. Poole, and G. Baxter, "Wavelength-selective reconfiguration in transparent agile optical networks," Proc. IEEE, vol. 100, no. 5, pp. 1056-1064, May 2012.

[3] “Draft revised g.694.1 version 1.3," Unpublished ITU-T Study Group 15, Question 6.

[4] W. Shi, Z. Zhu, M. Zhang, and N. Ansari, "On the effect of bandwidth fragmentation on blocking probability in elastic optical networks," IEEE Trans. Commun., vol. 61, no. 7, pp. 2970-2978, July 2013.

[5] A. Pages, J. Perello, S. Spadaro, and J. Comellas, "Optimal route, spectrum, and modulation level assignment in splitspectrum-enabled dynamic elastic optical networks," J. Opt. Commun. Netw., vol. 6, no. 2, pp. 114-126, Feb. 2014.

[6] A. Castro, F. Paolucci, F. Fresi, M. Imran, B. Bhowmik, G. Berrettini, G. Meloni, A. Giorgetti, F. Cugini, L. Velasco, L. Poti, and P. Castoldi, "Experimental demonstration of an active stateful PCE performing elastic operations and hitless defragmentation," in Proc. ECOC, Sept. 2013.

[7] S. Gringeri, N. Bitar, and T. Xia, "Extending software defined network principles to include optical transport," IEEE Commun. Mag., vol. 51, no. 3, pp. 32-40, Mar. 2013.

[8] A. Dixit, F. Hao, S. Mukherjee, T. Lakshman, and R. Kompella, "Towards an elastic distributed SDN controller," in Proc. ACM SIGCOMM, 2013, pp. 7-12.

[9] H. Iqbal, "A logically centralized approach for control and management of large computer networks," Ph.D. dissertation, University of Pittsburgh, 2012.

[10] M. Channegowda, R. Nejabati, and D. Simeonidou, "Softwaredefined optical networks technology and infrastructure: Enabling software-defined optical network operations [Invited]," J.Opt.Commun.Netw., vol.5, no. 10, pp.A274-A282, Oct. 2013.

[11] A. Giorgetti, F. Cugini, F. Paolucci, and P. Castoldi, "OpenFlow and PCE architectures in wavelength switched optical networks," in Proc. ONDM, Apr. 2012.

[12] M. Dallaglio, A. Giorgetti, N. Sambo, F. Cugini, and P. Castoldi, "Impact of slice-ability on dynamic restoration in GMPLS-based flexible optical networks," in Proc. OFC/ NFOEC, Mar. 2014.

[13] L. Liu, R. Munoz, R. Casellas, T. Tsuritani, R. Martinez, and I. Morita, "OpenSlice: An OpenFlow-based control plane for spectrum sliced elastic optical path networks," in Proc. ECOC, Sept. 2012.

[14] F. Cugini, G. Meloni, F. Paolucci, N. Sambo, M. Secondini, L. Gerardi, L. Poti, and P. Castoldi, "Demonstration of flexible optical network based on path computation element," J. Lightwave Technol., vol. 30, no. 5, pp. 727-733, Mar. 2012.

[15] A. Giorgetti, N. Sambo, I. Cerutti, N. Andriolli, and P. Castoldi, "Label preference schemes for lightpath provisioning and restoration in distributed GMPLS networks," $J$. Lightwave Technol., vol. 27, no. 6, pp. 688-697, Mar. 2009.

[16] A. Banerjee, J. Drake, J. Lang, B. Turner, D. Awduche, L. Berger, K. Kompella, and Y. Rekhter, "Generalized multiprotocol label switching: An overview of signaling enhancements and recovery techniques," IEEE Commun. Mag., vol. 39, no. 7, pp. 144-151, July 2001.

[17] J. Perello, S. Spadaro, F. Agraz, M. Angelou, S. Azodolmolky, Y. Qin, R. Nejabati, D. Simeonidou, P. Kokkinos, E. Varvarigos, and I. Tomkos, "Experimental demonstration of a GMPLSenabled impairment-aware lightpath restoration scheme," J. Opt. Commun. Netw., vol. 4, no. 5, pp. 344-355, May 2012.

[18] “OpenFlow switch specification 1.4.0," Oct. 2013 [Online]. Available: http://www.opennetworking.org/.

[19] N. McKeown, T. Anderson, H. Balakrishnan, G. Parulkar, L. Peterson, J. Rexford, S. Shenker, and J. Turner, "OpenFlow: Enabling innovation in campus networks," Apr. 2008. [Online]. Available: http://www.opennetworking.org.

[20] S. Das, G. Parulkar, N. McKeown, P. Singh, D. Getachew, and L. Ong, "Packet and circuit network convergence with OpenFlow," in Proc. OFC/NFOEC, Mar. 2010.

[21] V. Gudla, S. Das, A. Shastri, G. Parulkar, N. McKeown, L. Kazovsky, and S. Yamashita, "Experimental demonstration of OpenFlow control of packet and circuit switches," in Proc. OFC/NFOEC, Mar. 2010.

[22] S. Azodolmolky, R. Nejabati, E. Escalona, R. Jayakumar, N. Efstathiou, and D. Simeonidou, "Integrated OpenFlowGMPLS control plane: An overlay model for software defined packet over optical networks," in Proc. ECOC, Sept. 2011.

[23] D. Simeonidou, R. Nejabati, and M. Channegowda, "Software defined optical networks technology and infrastructure: Enabling software-defined optical network operations," in Proc. OFC/NFOEC, Mar. 2013.

[24] R. Casellas, R. Martinez, R. Munoz, R. Vilalta, L. Liu, T. Tsuritani, and I. Morita, "Control and management of flexi-grid optical networks with an integrated stateful path computation element and OpenFlow controller [Invited]," J. Opt. Commun. Netw., vol. 5, no. 10, pp. A57-A65, Oct. 2013.

[25] F. Paolucci, F. Cugini, N. Hussain, F. Fresi, and L. Poti, "OpenFlow-based flexible optical networks with enhanced monitoring functionalities," in Proc. ECOC, Sept. 2012, pp. 1-3.

[26] H. Y. Choi, L. Liu, T. Tsuritani, and I. Morita, "Demonstration of BER-adaptive WSON employing flexible transmitter/ receiver with an extended OpenFlow-based control plane," IEEE Photon. Technol. Lett., vol. 25, no. 2, pp. 119-121, Jan. 2013.

[27] L. Liu, D. Zhang, T. Tsuritani, R. Vilalta, R. Casellas, L. Hong, I. Morita, H. Guo, J. Wu, R. Martinez, and R. Munoz, "Field trial of an OpenFlow-based unified control plane for multilayer multigranularity optical switching networks," $J$. Lightwave Technol., vol. 31, no. 4, pp. 506-514, Feb. 2013.

[28] A. Giorgetti, F. Paolucci, F. Cugini, and P. Castoldi, "Fast restoration in SDN-based flexible optical networks," in Proc. OFC, Mar. 2014. 
[29] L. Tancevski, "SDN concept: From theory to network implementation," in Proc. OFC, Mar. 2014.

[30] S. Arakawa, Y. Kanitani, M. Murata, and K. Kitayama, "A wavelength assignment method for distributed wavelengthrouted networks using a circular wavelength-list," in Proc. BroadNets, Oct. 2005.

[31] A. Farrel, A. Iwata, N. Fujita, and G. Ash, "Crankback signaling extensions for MPLS and GMPLS RSVP-TE," IETF RFC 4920, July 2007.

[32] A. Giorgetti, N. Sambo, I. Cerutti, N. Andriolli, and P. Castoldi, "Suggested vector scheme with crankback mechanism in GMPLS-controlled optical networks," in Proc. ONDM, Feb. 2010.

[33] J. Dadap, P. B. Chu, I. Brener, C. Pu, C. D. Lee, K. Bergman, N. Bonadeo, T. Chau, M. Chou, R. Doran, R. Gibson, R. Harel, J. Johnson, S. S. Lee, S. Park, D. Peale, R. Rodriguez, D. Tong, M. Tsai, C. Wu, W. Zhong, E. Goldstein, L. Y. Lin, and J. Walker, "Modular MEMS-based optical cross-connect with large port-count," IEEE Photon. Technol. Lett., vol. 15, no. 12 , pp. $1773-1775$, Dec 2003.

[34] T. Zami, "High degree optical cross-connect based on multicast switch," in Proc. OFC, Mar. 2014.
[35] A. Giorgetti, F. Cugini, N. Sambo, F. Paolucci, N. Andriolli, and P.Castoldi, "Path state-based update ofPCEtraffic engineering database in wavelength switched optical networks," IEEE Commun. Lett., vol. 14, no. 6, pp. 575-577, June 2010.

[36] D. Alvarez, V. Lopez, J. Anamuro, J. de Vergara, O. de Dios, and J. Aracil, "Utilization of temporary reservation of path computed resources for multi-domain path computation element protocols in WDM networks," in Proc. NOF, Nov. 2011.

[37] R. Martinez, R. Casellas, and R. Munoz, "Experimental assessment of dynamic integrated restoration in GMPLS multilayer (MPLS-TP/WSON) networks," in Proc. ECOC, Sept. 2012.

[38] F. Paolucci, N. Sambo, F. Cugini, A. Giorgetti, and P. Castoldi, "Experimental demonstration of impairment-aware PCE for multi-bit-rate WSONs," J. Opt. Commun. Netw., vol. 3, no. 8, pp. 610-619, Aug. 2011.

[39] M. Tornatore, F. De Grandi, R. Muoz, R. Martinez, R. Casellas, and A. Pattavina, "Effects of outdated control information in control-plane-enabled optical networks with path protection," J. Opt. Commun. Netw., vol. 1, no. 2, pp. A194-A204, July 2009. 